\title{
La prevención de las arbovirosis desde el enfoque de la ciencia y la tecnología
}

\author{
The prevention of Arboviruses from the perspective of science and \\ technology
}

Haydeé Linares Sosa. ${ }^{1}$, María de los Ángeles Daniel. ${ }^{2}$, Omara Milian Zambrana. ${ }^{3}$ \& Lumey Mesa Rodríguez. ${ }^{4}$

Recibido: 05-02-2021 / Revisado: 14-02-2021 /Aceptado: 05-03-2021/ Publicado: 05-04-2021

\section{DOI: https://doi.org/10.33262/anatomiadigital.v4i2.1617}

\begin{abstract}
.
Introduction: Arboviruses are a group of febrile viral diseases transmitted by hematophagous insects (mosquitoes) that occur in tropical and subtropical areas and that constitute an important public health problem in countries of Africa, Asia and America. Objective: to establish the relevance of the prevention of arboviruses from the perspective of science and technology. Methodology: The scientific method based on the documentary analysis of the results of the
\end{abstract}

\section{Resumen.}

Introducción: Las arbovirosis son un conjunto de enfermedades víricas febriles transmitidas por insectos hematófagos (mosquitos) que se dan en áreas tropicales y subtropicales y que constituyen un importante problema de salud pública en países de África, Asia y América. Objetivo: fundamentar la pertinencia de la prevención de las arbovirosis desde el enfoque de la ciencia y la tecnología. Metodología: Se utilizó el método científico sustentado en la realización del

\footnotetext{
${ }^{1}$ Universidad de Ciencias Médicas de Matanzas, Facultad de Ciencias Médicas Juan Guiteras Gener,

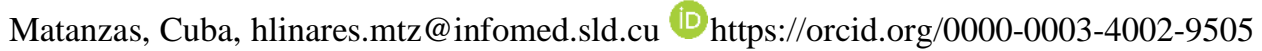

${ }^{2}$ Universidad de Ciencias Médicas de Matanzas, Facultad de Ciencias Médicas Juan Guiteras Gener, Matanzas, Cuba, mariadaniel.mtz@infomed.sld.cu (D) https://orcid.org/0000-0002-6781-9024

${ }^{3}$ Universidad de Ciencias Médicas de Matanzas, Facultad de Ciencias Médicas Juan Guiteras Gener, Matanzas, Cuba, omaramz.mtz@infomed.sld.cu (D) https://orcid.org/0000-0002-7254-765X

4 Centro Provincial de Higiene y Epidemiología, Matanzas, Cuba, lumey.mtz@infomed.sld.cu https://orcid.org/0000-0003-4706-109X
} 
investigations carried out by different authors on the prevention of arboviruses from the perspective of science and technology was used. Results: the development of science and technology in today's society play an important role in the prevention of arboviruses, maximum when the political will of the state and the high responsibility of researchers, form an inseparable pairing. Conclusions: science, technology and society constitute an important area of work in academic research, public policy and education, in order to understand the social aspects of the scientific and technological phenomenon of society.

Keywords: Arboviruses, prevention, science, technology and society análisis documental de los resultados de las investigaciones realizadas por diferentes autores acerca de la prevención de las arbovirosis desde el enfoque de la ciencia y la tecnología. Resultados: el desarrollo de la ciencia y la tecnología en la sociedad actual juegan un importante papel en la prevención de las arbovirosis, máxima cuando la voluntad política del estado y la elevada responsabilidad de los investigadores, forman un binomio inseparable. Conclusiones: la ciencia, tecnología y sociedad constituyen una importante área de trabajo en investigación académica, política pública y educación, en aras de entender los aspectos sociales del fenómeno científico y tecnológico de la sociedad.

Palabras claves: $\begin{gathered}\text { Prevención, } \\ \text { arbovirosis, ciencia, tecnología y } \\ \text { sociedad }\end{gathered}$

\section{Introducción.}

Las arbovirosis son un conjunto de enfermedades víricas febriles transmitidas por insectos hematófagos (mosquitos) que se dan en áreas tropicales y subtropicales y que constituyen un importante problema de salud pública en países de África, Asia y América. Existen unas 500 enfermedades producidas por arbovirus (López, Nozawa, \& Linhares, 2014) pero las que tienen mayor incidencia son fiebre amarilla, dengue, chicungunya y zika.

Estas enfermedades representan más del $17 \%$ de todas las enfermedades infecciosas, y provocan cada año más de 1 millón de defunciones. Más de 2500 millones de personas, en más de 100 países, corren el riesgo de contraer dengue. Cada año, el paludismo provoca más de 400000 defunciones en todo el mundo, la mayor parte de ellas entre niños menores de cinco años. Muchas de las enfermedades son prevenibles mediante medidas de protección fundamentadas en el actuar conductual tanto en el seno familiar como en el contexto social (Organización Mundial de la Salud, 2016), por lo que la escuela juega un rol fundamental, si tomamos en cuenta el papel que pueden desempeñar los estudiantes no solo a nivel familiar, sino también a nivel social, además de la importancia que para la solución de este importante problema de salud tienen la aplicación de la ciencia y la tecnología. 
La ciencia es uno de los productos más elevados de la actividad espiritual del hombre como resultado de la construcción de conceptos teoría y conocimientos en una disciplina particular. Formar la conciencia social, constituye un sistema histórico formado de conocimientos ordenados cuya veracidad se comprueba y se puntualiza en el curso de la práctica social.

En toda la historia de la humanidad, el hombre ha procurado garantizar y mejorar su nivel de vida mediante un mejor conocimiento del mundo que le rodea y un dominio más eficaz de él, es decir, mediante un desarrollo constante de la ciencia, entre ellas, las médicas. Gracias a la Revolución donde el Comandante en Jefe expresara el 15 de enero de 1960 "El futuro de Cuba tiene que ser necesariamente un futuro de hombres de ciencia" donde la ciencia y la tecnología están al servicio de toda la sociedad es que hemos alcanzado logros en la Salud Pública comparables con los países del primer mundo, a pesar del alto precio que hemos tenido que pagar por ello al ser Cuba un país bloqueado hace más de 50 años.

Hoy, la tecnología y la ciencia son parte del sistema de vida de todas las sociedades, se están uniendo a la voluntad social y política para controlar sus propios destinos, sus medios y el poder para hacerlo. Cuba no escapa de esta realidad y un ejemplo de ello es su aplicación en la erradicación de las arbovirosis.

El objetivo de este artículo se enfoca en fundamentar la pertinencia de la prevención de las arbovirosis desde el enfoque de la ciencia y la tecnología.

\section{Metodologia.}

Se utilizó el método científico sustentado en la realización del análisis documental de los resultados de las investigaciones realizadas por diferentes autores acerca de la prevención de las arbovirosis desde el enfoque de la ciencia y la tecnología.

La estrategia de búsqueda que se estableció fue la utilización de las palabras clave o descriptores, conectados por intermedio del operador booleano AND.

Las palabras clave utilizadas fueron "prevención and arbovirosis" y "ciencia and tecnología", siendo estas identificadas a través de DECs o de MeSH. De esta forma, fueron utilizados para la búsqueda los artículos referidos descriptores en idioma español, portugués e inglés. La búsqueda fue realizada en las bases de datos Scielo (Scientific Electronic Library Online), Science Direct y Google Académico, en el mes de septiembre de 2020.

Los criterios de inclusión para la selección de los artículos fueron: artículos en español, inglés y portugués disponibles en los portales de datos seleccionados que mostraban adherencia a la temática. Los criterios de exclusión fueron las investigaciones que se encontraron repetidas en las bases de datos los editoriales y las cartas al editor. 


\section{Resultados.}

Las enfermedades transmitidas por insectos vectores son complejas y dependen de la interacción de factores biológicos, ecológicos, sociales y económicos. Es decir, los determinantes de salud juegan un papel clave en la probabilidad de sufrir este tipo de enfermedades. En ausencia de vacunas (excepto para la fiebre amarilla), las estrategias de control vectorial junto con medidas preventivas personales pueden reducir la incidencia de estas enfermedades (Andersson, Nava, Arosteguí, Morales, Suazo, Legorreta, \& Cortés, 2015). Para alcanzar mejores resultados en el control vectorial, es necesaria una participación comunitaria activa y realizar actividades de promoción de la salud (Andersson, Nava, Arosteguí, Morales, Suazo, Legorreta, \& Cortés 2015; Kumar, Rathi, \& Goel, 2018), acciones estas que unidas al elevado desarrollo de la ciencia y la tecnología alcanzado por el país, logran un actuar permanente ante estas enfermedades. La autora asume que la ciencia como un producto general y espiritual del desarrollo social, es un elemento integrador de la cultura de la nación y está sujeta en sus limitaciones o en su expansión a las posibilidades que ofrece el modo de ser de la sociedad, además influyen en plenitud dimensional por las concepciones y los progresos de la universalidad de su carácter y el peculiar desarrollo interno de cada una, a lo que hay que añadir el papel influyente de la personalidad humana, es decir, del cultor de la ciencia, por lo que la responsabilidad social de cada individuo en aras de resolver todo lo relacionado con la prevención de las arbovirosis, con los beneficios de la ciencia y la tecnología es de suma importancia.

La ciencia parte de la conciencia social, constituye un sistema de conocimientos ordenados, que posibilitan la comprobación de su veracidad en el curso de la práctica social. Utiliza en su desarrollo los recursos del pensamiento lógico y formula sus conclusiones basándose en hechos. En ella tiene gran relevancia el conocimiento teórico, que surge mediante el ordenamiento de fenómenos conocidos y el descubrimiento de las relaciones entre ellos, las cuales se sistematizan mediante una ardua tarea de abstracción. Al abordar la misma como actividad humana, no solo se dedica a la producción de sistemas de conocimientos teóricos como resultado de los procesos de investigación, acerca de la naturaleza, la sociedad y el pensamiento, sino que precisa de la propagación de estos y de su aplicación para la transformación de la realidad; por lo que tiene como base la práctica histórico- social de la humanidad, aspecto este que adquiere gran valor ligado al componente educativo que presenta la acción de prevenir enfermedades como las arbovirosis.

Los principales representantes del marxismo - leninismo analizaron los problemas relacionados con la ciencia, su desarrollo, la adquisición de los conocimientos por el hombre desde puntos de vista filosóficos. Elaboraron la teoría marxista leninista sobre la naturaleza y la sociedad basándose en los adelantos científicos en su época y el empleo del método dialéctico para desentrañar la interrelación de los fenómenos naturales y sociales. 
La adquisición de profundos conocimientos científicos es la base para la formación en las nuevas generaciones de la concepción científica del mundo. Al respecto Lenin planteaba que “... la vieja enseñanza memorista y el viejo adiestramiento autoritario deben ser sustituidos por el arte de asimilar toda la suma de conocimientos humanos y asimilados de tal modo que el conocimiento no sea algo aprendido de memoria, sino algo pensado por nosotros mismos, como una conclusión que se impone necesariamente desde el punto de vista de la instrucción moderna”. (Lenin, 1961: 483).

A decir de la investigadora Casaña la ciencia es un fenómeno complejo que puede estudiarse desde diferentes perspectivas (Casaña, 2010) y refiere: “...no sólo como un sistema de conceptos, proposiciones, teorías, hipótesis, etcétera, sino también, simultáneamente, como una forma específica de la actividad social dirigida a la producción, distribución y aplicación de los conocimientos acerca de las leyes objetivas de la naturaleza y la sociedad. Aún más, la ciencia se (...) presenta como una institución social, como un sistema de organizaciones científicas, cuya estructura y desarrollo se encuentran estrechamente vinculados con la economía, la política, los fenómenos culturales, con las necesidades y las posibilidades de la sociedad dada". (Krober, 1986: 37)

En la actualidad el enfoque de los Estudios en Ciencia, Tecnología y Sociedad (CTS) permite adentrarnos en la ciencia respecto a sus características culturales, rasgos epistemológicos, éticos y su dimensión estética en su interacción con la sociedad, para comprender aspectos sociales del fenómeno científico por su heterogeneidad teórica, metodológica e ideológica en su estrecha interrelación con el fenómeno educativo y las ciencias y disciplinas que lo estudian. (Núñez, 2007)

La ciencia como una forma especial del conocimiento teórico, en las condiciones de la revolución científico- tecnológico contemporáneo se amplían y llegan a concretarse en la sociedad, convirtiéndose en una fuerza social directa.

La sociedad es el resultado de la acción recíproca de los hombres en la historia de la producción de bienes materiales y la producción y reproducción de su vida espiritual. En el modo de producción dentro de la sociedad se debe tener en cuenta las fuerzas productivas, dentro de las mismas aparecen, de una parte, los medios de producción y la fuerza de trabajo, donde se evidencia la relación ciencia y tecnología. (García, 2010)

Al estudiar los beneficios de la ciencia, no se trata solamente de los efectos en la sociedad actual, sino también en la futura. En las sociedades ancestrales estaban bien definidas las funciones del individuo, había una armonía entre la naturaleza, la sociedad y el hombre. Ahora bien, la ciencia trajo consigo la desaparición de este marco tradicional, la ruptura del equilibrio entre el hombre y la sociedad y una profunda modificación del ambiente; pero de ello no se debe culpar directamente a la ciencia. Los progresos de la ciencia han sido muy rápidos en los países desarrollados; en cambio, en los subdesarrollados su 
adquisición es tan lenta, que cada día la diferencia entre ellos se hace más grande, lo cual agrava la situación de dependencia de los subdesarrollados con respecto a los desarrollados.

La influencia de la ciencia y la tecnología sobre la vida del ser humano es compleja, porque es determinante para el proceso de su desarrollo, sus efectos se extienden hacia numerosos aspectos relacionados directa o indirectamente en el nivel de vida de la población, sin embrago el desarrollo desproporcionado y la brecha existente entre los países desarrollados y subdesarrollados, hace que este importante componente de la calidad y el nivel de vida sea una falacia para una gran parte de la población mundial. Por tanto, podemos decir que la Revolución Científico Técnica, tanto por su influencia sobre el desarrollo social en general como sobre el desarrollo de las ciencias médicas, ha representado una posibilidad extraordinaria de progreso para el proceso salud enfermedad en el hombre.

Eso se concreta en la mayoría de los indicadores como el aumento de las expectativas de vida, la sensible disminución de la morbilidad de enfermedades infecciosas en los países desarrollados y las enormes posibilidades que tienen a mano estas ciencias que no las tenían en el siglo pasado. Esto es indiscutible y es una cuestión de principios que no solo está ligada a la revolución científico técnica y su influencia sobre la medicina, sino a la influencia de ella sobre el desarrollo social en general. (Alfonso, Gómez, Galván, Alfonso, Villamil, 2013)

La ciencia y la tecnología son procesos sociales profundamente marcados por la civilización donde han crecido; el desarrollo científico y tecnológico requiere de una estimación cuidadosa de sus fuerzas motrices e impactos, un conocimiento profundo de sus interrelaciones con la sociedad. (Núñez, 2007)

La Medicina, una rama de las Ciencias Médicas, es un fenómeno en constante desarrollo donde se actualizan los conocimientos que son verificados en la práctica diaria, en un entorno social donde se orienta a la curación de enfermedades para mejorar la calidad de vida del hombre. La medicina es capaz de describir, explicar, pronosticar, transformar y en su caso particular curar las enfermedades valiéndose de la tecnología, investigación cuyo producto principal es los avances de la tecno-ciencia y la voluntad política del país y su perseverante estrategia para mantener en niveles bajos la infestación del agente trasmisor y también al desarrollo de un impresionante sistema médico preventivo y científico - investigativo, a la permanente educación higiénico sanitaria de la población, a la adquisición de medios de detección, y a la generación propia de métodos y tecnologías. Que la enfermedad no se convierta en endémica, es una decisión firme. Sin embargo, los episodios de introducción, debido a las constantes migraciones de personas desde el extranjero y a la colaboración cubana -una tendencia que continuará, son una amenaza latente. 
Los resultados en el sector de la salud, al que se suma el creciente desarrollo científicotécnico con el auge de la biotecnología, hacen de Cuba una potencia médica y científica. Las políticas de salud de nuestro gobierno le han permitido ser el único país latinoamericano capaz de controlar enfermedades como el dengue. Por eso es Cuba un ejemplo para el resto de las naciones del mundo. (Lemus, 2006)

Como la ciencia ha pasado a formar parte de las fuerzas productivas en mayor medida que antes, se considera hoy un agente estratégico del cambio en los planes de desarrollo económico y social. La ciencia ha llegado al punto de influir sobre la mentalidad de la humanidad.

La sociedad de hoy no está cautiva en las condiciones pasadas o presentes, sino que se orienta hacia el futuro. La ciencia no es simplemente uno de los varios elementos que componen las fuerzas productivas, sino que ha pasado a ser un factor clave para el desarrollo social, que cala cada vez más a fondo en los diversos sectores de la vida.

La ciencia trata de establecer verdades universales, un conocimiento común sobre el que exista un consenso y que se base en ideas e información cuya validez sea independiente de los individuos. Hay algo que es de gran importancia resaltar, y es que el rol de la ciencia en la sociedad es inseparable del de la tecnología.

Hoy, la tecnología y la ciencia son parte del sistema de vida de todas las sociedades, uniéndose a la voluntad social y política para controlar sus propios destinos, sus medios y el poder para hacerlo. Cuba no escapa de esta realidad y un ejemplo de ello es su aplicación a la erradicación de las arbovirosis.

Una característica del momento actual es la conexión indisoluble, interacción y el acondicionamiento mutuo de la sociedad con la ciencia. Ella es esencial para el desarrollo social y cada vez adquiere un carácter más masivo. Por la voluntad política del país, por el trabajo intersectorial, el que debe seguir incrementándose para solucionar los riesgos que aún persisten, por la participación de la comunidad organizada y por la aplicación de derechos a los que incumplen en toda la historia de la humanidad, el hombre ha procurado garantizar y mejorar su nivel de vida mediante un mejor conocimiento del mundo que le rodea y un dominio más eficaz de él, es decir, mediante un desarrollo constante de la ciencia, entre ellas, las de la educación en la sociedad.

La tecnología no solamente invade toda la actividad industrial, sino también participa profundamente en la humana, en todos sus campos de actuación. El hombre moderno utiliza en su comportamiento cotidiano, y casi sin percibirlo, una inmensa avalancha de contribuciones de la tecnología: A pesar de que existe conocimiento que no puede ser considerado como tecnológico, este también lo es, porque a pesar de su origen, es utilizado en el sentido de transformar elementos materiales _materias primas, componentes, etc., o simbólicos -datos, información, etc.- en bienes o servicios, para 
modificar su naturaleza o sus características. Cuba utiliza tecnologías en el enfrentamiento a las arbovirosis como son los tratamientos con adulticidas, abaticidas y fungicidas.

La Organización Mundial de la Salud (OMS) declaró que las medidas de control vectorial son fundamentales para lograr y mantener la reducción de las arbovirosis (World Health Organization, 2012b). Las creencias de salud de las personas, sus conocimientos y las actitudes relacionadas con el dengue influyen en sus prácticas y comportamientos de salud (Wong, Shakir, Atefi, \& AbuBakar, 2015) por ello es esencial que su conocimiento esté basado en la evidencia científica. Esto se hace extensible a las demás arbovirosis transmitidas por mosquitos: fiebre amarilla, Chikungunya y Zika.

Fortalecer las acciones de ordenamiento ambiental, principalmente la eliminación de criaderos del vector en cada domicilio y en áreas comunes de los barrios y ciudades (parques, escuelas, cementerios, etc.). Es parte del medio ambiente y es hacer ciencia. Aplicar medidas para el control de criaderos con la utilización de métodos físicos, biológicos y químicos, en las que participen activamente la familia y la comunidad. Forma parte de la tecnología.

Determinar las zonas de alto riesgo de transmisión (estratificación de riesgo) y dar prioridad a aquellas donde existan concentraciones de personas (escuelas, terminales de transporte, hospitales, centros de salud, etc.). En esas instalaciones deberá eliminarse la presencia del mosquito en un radio de al menos 400 metros a la redonda. Es hacer ciencia. Mantener el equipo de fumigación en buen funcionamiento y utilizarlo adecuadamente es parte de la tecnología.

Las organizaciones comunitarias, estructuras de salud y de gobierno, con un adecuado análisis de la situación de partida y el contexto social, requieren de una propuesta lo suficiente flexible y adaptable y de una metodología que facilite la expresión de la diversidad local aplicándolo a la ciencia y la tecnología en la sociedad.

Todo lo anterior da la medida de cuanto hay que hacer, y de cuanto hay que modificar en métodos de trabajo y enseñanzas, y que deben particularizarse a cada población específica, según sus patrones culturales, hábitos y costumbres. Para aplicar la educación para la salud es necesario usar una metodología adecuada que incorpore métodos de aprendizaje activos, que vayan dirigidos hacia las influencias sociales y la de los medios de comunicación de masas, que refuerce los valores individuales y las normas grupales, que promueva, fundamentalmente, el desarrollo de habilidades sociales.

Las estrategias de intervención consisten en identificar y organizar un conjunto de medidas sanitarias aplicadas a una persona, grupo o comunidad, que implican acciones de promoción, mantenimiento y restauración de la salud, que deben tener como base la intersectorialidad, indispensable para su realización. Sin lugar a dudas, la solución para 
transitar de la enfermedad al bienestar, menos costosa y más segura, a la vez es en sí un problema, porque no siempre es valorada de esta forma por todos los que tienen que ver con su ejecución y porque cuando es concebida de manera clara, aún en su forma de materialización, encuentra diversos obstáculos derivados de formas de pensar que no se han modificado a la misma velocidad que requiere la transformación.

A continuación veremos como la tecnología y la ciencia contribuyen a mejorar el estado de salud de la población en relación a las arbovirosis, donde el doctor Gerardo Castellanos, jefe de la unidad de Enfermedades Desatendidas, Transmitidas por vectores y Tropicales de las organizaciones Panamericana y Mundial de la Salud (OPS /OMS) en Washington, elogió la labor de Cuba en la prevención y control del Dengue, considerada una de las arbovirosis más frecuentes del país.(Castro, Pérez, Pérez, Polo, \& López, 2011)

Durante el XIV Curso Internacional sobre Dengue celebrado en el Instituto de Medicina Tropical Pedro Kourí (IPK), en la capital, con profesionales de 35 países, el experto dijo a la Agencia Internacional de Noticias (AIN) que esa institución es uno de los grandes bastiones y en la que más se respalda ese organismo para trabajar en el control de la enfermedad en las Américas.

Cuba desarrolla dos proyectos de vacunas contra el dengue con resultados alentadores, aunque hoy lo más importante es controlar el aedes aegypti, el mosquito transmisor del virus, señaló la doctora María Guadalupe Guzmán. Detalló que se busca un compuesto tetravalente, contra los cuatros serotipos del virus del dengue, y que además ofrezca protección tanto a quienes viven en áreas endémicas, como a los que viajan a esos lugares. (Guzman, Kouri, \& Halstead, 2017), además desarrolla un amplio programa de investigaciones relacionadas con estas enfermedades, entre estas se encuentran las lideradas por el Instituto de Medicina Tropical Pedro Kourí, se destacan el establecimiento de la hipótesis integral sobre el dengue hemorrágico y los estudios virológicos para la caracterización de los virus causantes de las epidemias y los brotes de esta enfermedad en Cuba, y los marcadores de virulencia y su asociación con el dengue grave. Estas investigaciones también han beneficiado a otros países de la Región.

Los estudios entomológicos y las investigaciones operacionales para el control del mosquito aedes aegypty han permitido caracterizar e identificar los principales sitios de cría y los mecanismos de resistencia del vector. Asimismo, BioCubaFarma y otros centros trabajan para la obtención de un candidato vacunal contra esta enfermedad. También, son relevantes los estudios sobre los determinantes socioeconómicos y la participación social e intersectorial, así como los relacionados con los conocimientos, las actitudes y las prácticas de la población. (Rojo, et al 2018)

La Organización Panamericana de la Salud/Organización Mundial de la Salud (OPS/OMS) y el Ministerio de Salud de Costa Rica crearon "Pueblo Pitanga. Enemigos 
Silenciosos", un juego virtual para PC que busca educar y generar conciencia en los niños y jóvenes sobre el dengue y la importancia de eliminar los criaderos del mosquito que transmite la enfermedad.

"Con esta iniciativa utilizamos la tecnología y el entretenimiento para educar", señaló el representante de la OPS/OMS en Costa Rica, Jorge Prosperi, y manifestó que "los chicos son grandes agentes de cambio que pueden contribuir a eliminar los criaderos del mosquito, la solución más importante para controlar y prevenir el Dengue". (OMS/OPS, 2003)

Mosquitos machos modificados genéticamente muestran potencial para controlar la propagación del dengue, difundió la revista Nature Biotechnology en su edición más reciente.

Descendientes de los insectos modificados genéticamente llegan a la fase de larva, pero no sobrepasan la de pupa, lo que permite evitar una mayor propagación de la enfermedad, explicaron expertos de la empresa biotecnológica Oxitec, de la Universidad de Oxford.

María Guadalupe Guzmán, Jefa del Departamento de Virología del Instituto de Medicina Tropical "Pedro Kourí" (IPK), en La Habana, señaló que, en esta propuesta realizada a partir de estudios desarrollados a nivel mundial, Cuba tuvo una importante participación, con un gran impacto por su trabajo.

Aunque la ciencia y la tecnología aportan elementos decisivos para la lucha contra estas enfermedades sin el apoyo de la comunidad y la escuela es imposible prevenir las mismas. Álvarez (2017) plantea que la escuela es un agente primario de socialización en el cual confluyen estudiantes, profesores y familiares; convirtiéndose en un medio idóneo para esta labor, vista de manera holística, o sea, como un proceso en el que intervienen tanto la escuela, la familia y la comunidad.

Se asume este criterio y se concuerda con Carvajal, Torres, Pascual, Justiz, \& Lòpez (2016), cuando plantean que "Las instituciones educacionales están llamadas a coordinar los esfuerzos y acciones alrededor de los problemas identificados, para de conjunto con los miembros de la comunidad, promover mejores estilos de vida, normas más adecuadas de comportamiento, satisfacer carencias en aspectos educacionales, sociales, culturales, ecológicos, ambientales y de salud, tienen la posibilidad de convertirse en el centro de mayor influencia cultural de la zona donde se encuentra enclavada.

El Estado como responsable de políticas públicas educativas, de salud, de medio ambiente, debe estar comprometido en todos sus niveles de gobierno (nacional, provincial y municipal), con las necesidades de la comunidad, para apoyar, promover, facilitar y comunicar todo aquello que implica participación social en la solución de problemas comunes. Prevenir implica conocimiento y responsabilidad ambiental. En Cuba, la voluntad política del estado cubano, unido a la conciencia social de nuestro pueblo y a la 
dimensión subjetiva del sistema de valores de la sociedad, han hecho posible que se trabaje incesantemente en la prevención de las arbovirosis como problema de salud presente en estos tiempos.

La base del cambio es, sin duda, la educación y adquisición de hábitos ambientales preventivos desde edades tempranas. Para ello es recomendable la participación activa del conjunto de educadores de todas las disciplinas del conocimiento.

Los presupuestos teóricos que sustentan la política educativa cubana expresan la inmensa posibilidad de la educabilidad del hombre en el contexto social donde se desarrolla. Dentro de la psicología de orientación dialéctica materialista, el término personalidad adquiere una doble significación como categoría psicológica, y esto facilita el mejoramiento de la calidad en la educación. Si las aplicaciones de las concepciones materialistas de la personalidad son sustentadas básicamente en la teoría de Vigostky acerca de la concepción histórico social de las funciones psíquicas superiores, se considera que en el proceso de desarrollo histórico, el hombre social cambia los modos y procedimientos de su conducta, crea códigos nuevos, establece estilos de vida acordes al proceso histórico social concreto, por tanto en el ser humano existe una relación entre lo interno y lo externo pero que en la actividad del estudiante se hace imposible lograr sin la comunicación social.

\section{Conclusiones.}

- En el contexto actual, la ciencia, tecnología y sociedad constituyen una importante área de trabajo en investigación académica, política pública y educación; en aras de entender los aspectos sociales del fenómeno científico y tecnológico de la sociedad. Al tener en cuenta este enfoque y aplicándolo al enfrentamiento a las arbovirosis, lograremos un rotundo éxito en la erradicación de estas enfermedades, a la vez que se cultiva la responsabilidad social de los actores implicados con el desarrollo científico- tecnológico alcanzado en una sociedad que mantiene entre sus premisas una férrea voluntad política de preservar la salud de la población y elevar la calidad de vida.

\section{Referencias bibliográficas.}

Alfonso Pérez OA, Gómez Verano MR, Galván Noa T, Alfonso Martínez PA, Villamil Fumero K (2013). La educación en ciencia, tecnología y sociedad: su importancia en la Educación Médica Superior. Mediciego 2013; 19 (1). Recuperado de: https://www.medigraphic.com/cgi-bin/new/resumen.cgi?IDARTICULO=43259

Álvarez Rivera, C. (2017). Importancia de la socialización en el aula en el proceso docente. Medellín: Facultad de Educación. 
Andersson, N., Nava Aguilera, E., Arosteguí, J., Morales Pérez, A., Suazo Laguna, H., Legorreta Soberanis, J., \& Cortés Guzmán, AJ. (2015). Evidence based community mobilization for dengue prevention in Nicaragua and Mexico (Camino Verde, the Green Way): cluster randomized controlled trial. Bmj, 351, h3267.

Carvajal Rodríguez, C., Torres Cueto, M.A., Pascual Betancourt, P., Justiz Charón, E. \& Lòpez Gomez, AB. (2016). Prevención integral y Promoción de la Salud en la Escuela. La Habana: Ministerio de Educación.

Casaña Díaz, M. (2010).Texto complementario para el postgrado de Problemas sociales de la Ciencia y la Tecnología: la relación Filosofía-Ciencia desde una perspectiva Marxista-Leninista. Universidad de Ciencias Pedagógicas "Juan Marinello". Matanzas.

Castro M, Pérez D, Pérez K, Polo V, \& López L. (2011). Contextualización de una estrategia comunitaria integrada para la prevención del dengue. Revista Cubana Medicina Tropical 60(1). Recuperado de: http://scielo.sld.cu/scielo.php?script=sci_arttext\&pid=S037507602008000100013\&lng=es

García Batista G. (2010) Programa de la Maestría en Ciencias de la Educación. Instituto Pedagógico Latinoamericano y Caribeño, Cuba. Curso: Temas actuales de la política, la ideología, la ciencia, la tecnología y la cultura. Ed. Pueblo y Educación. Ciudad de la Habana, p. 29 y 30.

Guzman MG, Kouri G, Halstead S. (2017) Do escape mutants explain rapid increases in dengue case-fatality rates within epidemics? The Lancet 2000; 355: 1902-1903. (Do escape mutants explain rapid increases in dengue case-fatality rates within epidemics? Recuperado de: https://pubmed.ncbi.nlm.nih.gov/10866460/

Krober G., (1986) "Acerca de las relaciones entre la historia y la teoría del desarrollo de las ciencias". En: Revista Cubana de Ciencias Sociales, año IV, no. 10, enero-abril. La Habana, p.37

Kumar, V., Rathi, A., Lal, P., \& Goel, S. K. (2018). Malaria and dengue: Knowledge, attitude, practice, and effect of sensitization workshop among school teachers as health educators. Journal of family medicine and primarycare, 7(6), 1368.

Lemus Pérez G. (2006). Dengue, un problema social reemergente en América Latina. Estrategia para su erradicación, Biotecnología Aplicada, 23(2). Recuperado de: https://elfosscientiae.cigb.edu.cu/PDFs/Biotecnol\%20Apl/2006/23/2/BA002302E N130-136.pdf

Lenin, VI. (1961). Sobre el significado del materialismo militante. Obras escogidas Tomo III. p 483. Editorial Progreso, Moscú. 
Lopes, N., Nozawa, C., \& Linhares, R. E. C. (2014). Características gerais e epidemiologia dos arbovírus emergentes no Brasil. Revista Pan-Amazônica de Saúde, 5(3), 55-64. Recuperado de: https://doi: 10.5123/S217662232014000300007.

Núñez Jover, J. (2007). La Ciencia y la Tecnología como procesos sociales. Lo que la educación científica no debería olvidar. La Habana: Félix Varela.

Organización Mundial de la salud (2016). Enfermedades transmitidas por vectores.

Organización Mundial de la Salud/Organización Panamericana Salud/Center Disease Control. (2003). La implementación del Dengue Net en las Américas. Puerto Rico. Recuperado de: https://www.who.int/csr/resources/publications/dengue/en/whocdscsrgar20038sp. pdf

Rojo Pérez, N., Valenti Pérez, C., Martínez Trujillo, N., Morales Suárez, I., Martínez Torres, E., Fleitas Estévez, I., et al. (2018). Ciencia e innovación tecnológica en la salud en Cuba: resultados en problemas seleccionados. Revista Panamericana Salud Publica, 42: e32. Recuperado de:https://doi.org/10.26633/RPSP.2018.32

Wong LP, Shakir SM, Atefi N, \& AbuBakar S. (2015). Factors affecting dengue prevention practices: nationwide survey of the Malaysian public. PLoS One; 10: e0122890. Recuperado de: http://dx.doi.org/10.1371/ journal. pone.0122890

World Health Organization. (2012b). Global strategy for dengue prevention and control 2012-2020.WHO/Department of Control of Neglected Tropical Diseases WHO/HTM/NTD/VEM/2012.5.

\section{LCiencia}




\section{PARA CITAR EL ARTÍCULO INDEXADO.}

Linares Sosa, H., Daniel, M. de los Ángeles, Milian Zambrana, O., \& Mesa Rodríguez, L. (2021). La prevención de las arbovirosis desde el enfoque de la ciencia y la tecnología . Anatomía Digital, 4(2), 54-67. https://doi.org/10.33262/anatomiadigital.v4i2.1617

\section{\Ciencia}

El artículo que se publica es de exclusiva responsabilidad de los autores y no necesariamente reflejan el pensamiento de la Revista Anatomía Digital.

El artículo queda en propiedad de la revista y, por tanto, su publicación parcial y/o total en otro medio tiene que ser autorizado por el director de la Revista Anatomía Digital.
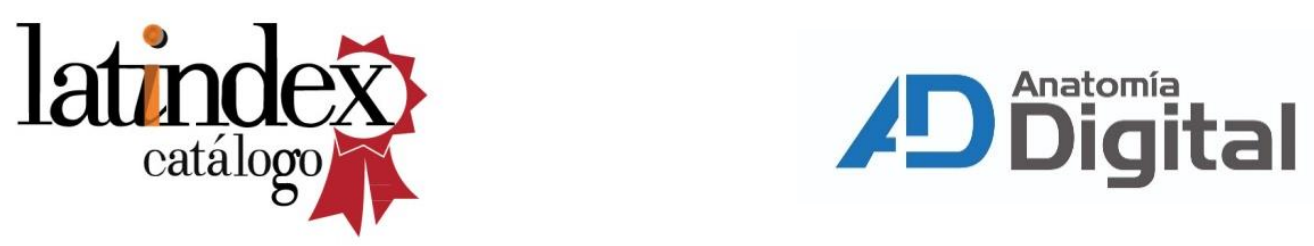